\title{
Remodelación cardiaca en pacientes con infarto de miocardio secundario a herida penetrante de corazón
}

\author{
Cardiac remodeling in patients with myocardial \\ infarction secondary to penetrating heart wound
}

\author{
Juan Manuel Senior, Clara Saldarriaga, Claudia María Navas, \\ Luz Helena lugo, Sergio Daniel Ortiz, Juan Carlos Parra • \\ Medellín (Colombia)
}

\section{Resumen}

Introducción: la frecuencia del trauma cardiaco se ha incrementado en los últimos años, se desconoce el mecanismo de remodelación miocárdica en estos pacientes.

Objetivos: evaluar la remodelación cardiaca en pacientes con herida penetrante de corazón comparando dos grupos, con y sin infarto de miocardio traumático asociado.

Metodología: estudio observacional, tipo cohorte y prospectivo. La cohorte expuesta fueron pacientes mayores de 12 años con herida de corazón e infarto traumático y los no expuestos no tuvieron infarto. En el periodo posoperatorio y seis meses después se realizó una evaluación clínica, ecocardiográfica y funcional.

Resultados: se incluyeron 31 pacientes de sexo masculino y dos de sexo femenino, con edad promedio de 31 años. Más de $90 \%$ de las heridas fueron por arma cortopunzante. La fracción de eyección y la fracción de acortamiento al inicio fueron significativamente menores en los pacientes con infarto traumático. La única variable con diferencia significativa entre los dos grupos fue la fracción de acortamiento (30.18 Vs. 34.72, p < 0.05). Seis meses después ambos grupos mejoraron la capacidad funcional, el grosor relativo de la pared disminuyó. Los pacientes con infarto tuvieron más probabilidad de desarrollar hipertrofia excéntrica ( $\mathrm{RR}$ : $2.67 \mathrm{IC}=0.72-9.89$ ). Las demás variables ecocardiográficas fueron similares al inicio y a los seis meses en ambos grupos.

Conclusión: los pacientes con infarto traumático desarrollaron hipertrofia excéntrica. A pesar de la demostración de un patrón claro de remodelación ventricular, las diferencias son pequeñas debido al número de pacientes y al corto tiempo de seguimiento. (Acta Med Colomb 2012; 37: 6-13).

Palabras clave: infarto de miocardio, traumático, remodelación, ecocardiografía.
Dr. Juan Manuel Senior Sánchez: Cardiólogo Hemodinamista, Professor Asociado de la Sección de Cardiología Universidad de Antioquia, Jefe de la Clínica de Falla Cardiaca y el Programa de Trasplante del Hospital Universitario San Vicente de Paúl, FACP; Dra. Clara Saldarriaga: Cardióloga, Internista, Profesora de la Sección de Cardiología de la Universidad de Antioquia; Dres. Claudia María Navas, Luz Helena Lugo, Sergio Daniel Ortiz y Juan Carlos Parra: Profesores del Servicio del Programa de Rehabilitación Cardiaca, Hospital San Vicente de Paúl. Medellín (Colombia). Correspondencia. Dr. Juan Manuel Senior, Hospital Universitario San Vicente de Paúl, Universidad de Antioquia, Sección Cardiología. Medellín (Colombia).

E-mail: mmbt@une.net.co Recibido: 03/II/2011 Aceptado: 06/X/2011 
Conclusions: patients with penetrating heart wounds with and without infarction showed a tendency to develop a remodelling process with eccentric hypertrophy. The differences encountered, however, are not significant and a longer follow-up is required, as well as a larger number of patients. (Acta

Med Colomb 2012; 37: 6-13).

Key words: myocardial infarction, traumatic, remodelling, echocardiography

\section{Introducción}

La frecuencia del trauma cardiaco se ha incrementado en forma significativa en los últimos años. En un hospital de la ciudad de Boston, la incidencia anual de heridas penetrantes del corazón pasó de 2.8 casos por año durante el periodo de 1956-1964 a ocho casos por año de 1965-1976. En Houston, un análisis de 30 años sobre 4459 pacientes con lesiones cardiovasculares demostró un incremento de 27-213 pacientes por año entre 1958 y $1988(1,2)$. En Colombia, esta tendencia se ha mantenido; el incremento de la violencia y las dificultades socioeconómicas han aumentado la frecuencia del trauma de tórax y la incidencia de herida penetrante de corazón. En un estudio realizado en el Hospital Universitario San Vicente de Paúl (HUSVP) de Medellín entre julio 1o. de 1990 y diciembre 31 de 1994, 1022 pacientes fueron intervenidos, lo anterior da un promedio de 227 pacientes intervenidos por año, cifra que supera en casi cinco veces, la reportada en la literatura mundial (3). La mayoría de las series reportan cifras de mortalidad que oscilan entre $5.5 \mathrm{y}$ $57.6 \%$ para los pacientes que llegan al hospital con signos vitales y son intervenidos (4-7).

En el seguimiento de estos pacientes pueden presentarse diversas complicaciones tales como isquemia e infarto agudo de miocardio, defectos en el séptum ventricular, pericarditis, empiema, sepsis y falla cardiaca (8-10). Los estudios disponibles en la literatura reportan cifras para infarto de miocardio que oscilan entre 3.3 y $9.1 \%(11,12)$. En el estudio realizado por Duque y colaboradores, se encontró durante la evaluación posoperatoria inicial infarto de miocardio documentado por hallazgos en el electrocardiograma (ECG) en $9,1 \%$ de estos pacientes, sin que se hubiera sospechado durante la intervención quirúrgica (3).

El proceso de remodelación cardiaca puede ser desencadenado, aun por eventos iniciales leves que no causan disfunción cardiaca inmediata, como pequeños infartos de miocardio $(13,14)$, y aunque se considera un proceso adaptativo, puede llegar a ser maladaptativo y producir cambios progresivos en la estructura cardiaca $(15,16)$; deterioro en la función miocárdica, insuficiencia cardiaca, discapacidad y muerte, aún sin que se presenten nuevos eventos (17 -23).

Después del infarto agudo de miocardio (IAM) no traumático, los segmentos infartados y no infartados del ventrículo izquierdo presentan cambios en la forma, el tamaño y el grosor (24-26). Este proceso se define como remodelación cardiaca y generalmente precede a la aparición de signos clínicos evidentes de falla cardiaca en los meses o años siguientes al infarto (27-29).
En resumen, las recientes publicaciones sugieren que la remodelación de la MEC durante la cicatrización después del IAM intenta restaurar la fuerza mecánica y la resistencia a la distensión en las zonas infartada y no infartada del ventrículo. Las estrategias futuras que busquen proteger la MEC en la zona infartada podrían contribuir a disminuir la mortalidad y morbilidad en los pacientes con IAM $(28,29)$.

Hasta el momento se desconoce el mecanismo causal del infarto de miocardio traumático y cómo es el proceso de remodelación cardiaca que desarrollan. Para aclarar este interrogante se decidió caracterizar y cuantificar el proceso de remodelación cardiaca que se presenta en los pacientes que desarrollan infarto de miocardio secundario a herida penetrante de corazón y compararlo con el que se presenta en los pacientes que no desarrollan infarto de miocardio.

\section{Material y métodos}

Se realizó un estudio observacional, analítico, tipo cohorte y prospectivo. La población de estudio correspondió a los pacientes que ingresaron al servicio de urgencias entre enero de 2002 y abril de 2004, por presentar herida penetrante de corazón. La cohorte expuesta comprendió todos los pacientes mayores de 12 años, procedentes del área metropolitana de Medellín a quienes se realizó toracotomía de urgencia y en cuyo posoperatorio se documentó infarto agudo de miocardio. Se diagnosticó infarto de miocardio teniendo en cuenta los siguientes criterios: 1) Cambios ECG compatibles con infarto de miocardio (Ondas Q patológicas definidas por una amplitud superior a $1 / 4$ de la amplitud del complejo QRS y una duración de 40 ms o más, que aparecen en dos o más derivaciones de un área coronaria definida, con elevación del segmento ST de $0,2 \mathrm{mV}$ de V1 a V3 o de $0.1 \mathrm{mV}$ en las otras derivaciones) que se identificaron en el posoperatorio (24-72 horas). Cuando los cambios sugerían infarto de cara inferior, se procedió a realizar derivaciones precordiales derechas para identificar infarto del ventrículo derecho asociado, y/o 2) Cambios ecocardiográficos caracterizados por anormalidades en el movimiento de la pared ventricular (hipocinesia o acinesia) que se identificaron durante las primeras tres semanas del posoperatorio (30). La cohorte no expuesta se conformó por los pacientes mayores de 12 años, procedentes del área metropolitana de Medellín con toracotomía por herida penetrante de corazón y en quienes no se logró documentar infarto del miocardio en el posoperatorio.

Los siguientes fueron criterios de exclusión: antecedente de cardiopatía hipertensiva, infarto previo, cardiomiopatía hipertrófica, fiebre reumática con compromiso valvular, 
cardiopatías congénitas incluyendo prolapso de la válvula mitral, comunicaciones interauriculares o interventriculares y consumo previo de medicamentos que puedan interferir con el proceso de remodelación cardiaca (IECA; antagonista de receptores AT2).

El cálculo del tamaño de la muestra se realizó mediante el programa EpiInfo Versión 6.04c teniendo en cuenta los siguientes parámetros: poder de $80 \%$, nivel de confianza de $95 \%$, prevalencia de remodelación cardiaca en el grupo expuesto de $50 \%$, prevalencia de remodelación cardiaca en el grupo no expuesto de 5\%, con una relación de expuesto: no expuesto de 1 a 2 ; se calculó un total de 13 pacientes para formar la cohorte expuesta y 26 para la cohorte no expuesta.

Para la conformación de la cohorte expuesta se incluyeron todos los pacientes que cumplían con los criterios en forma sucesiva. Para la conformación de la cohorte no expuesta se realizó un muestreo por conveniencia. Se seleccionaron como controles dos de cinco pacientes que seguían consecutivamente al caso teniendo en cuenta la edad más o menos 10 años.

Todos los pacientes firmaron el consentimiento informado previo a la participación en la investigación y con la suficiente ilustración y resolución de interrogantes por parte de los investigadores. El procedimiento de evaluación y seguimiento de los pacientes vinculados en el estudio fue el siguiente: en el posoperatorio y seis meses después cada paciente se evaluó por uno de los investigadores quien diligenció el formulario con los datos de identificación, las variables epidemiológicas (la edad, el sexo, la procedencia, los antecedentes, el tipo de arma) y las variables clínicas y paraclínicas (la localización de la lesión, las complicaciones quirúrgicas, la frecuencia cardiaca y el tipo de ritmo cardiaco). Además, durante la hospitalización se les realizó a todos los pacientes un ecocardiograma transtorácico y una evaluación funcional. En los pacientes con infarto el ecocardiograma se realizó durante las tres semanas siguientes del posoperatorio, dependiendo de la situación clínica del paciente entre dos y 16 días después de la herida cardiaca; la ecocardiografía y la evaluación funcional de los seis meses se realizaron en promedio a los 221 (184-303) días después de la herida. En los pacientes sin infarto la primera ecocardiografía se realizó en promedio a los 8 (2-40) días después de la herida cardiaca; la ecocardiografía y la evaluación funcional de seguimiento se realizaron en promedio a los 206 (183-269) días después de la herida. Las ecocardiografías se realizaron en un equipo Hewlett-Packard Sonus 5500, en el laboratorio de ecocardiografía de la unidad de cardiología, el cual cumple con las normas de la Sociedad Americana de Cardiología y el American College of Cardiology (ACC). Cada examen fue leído en forma independiente por dos cardiólogos y las discrepancias se resolvieron por consenso. Las variables ecococardiográficas que se tuvieron en cuenta fueron: el diámetro diastólico del ventrículo izquierdo (DDVI), el diámetro sistólico del ventrículo izquierdo (DSVI), el área de la aurícula derecha (AAD), el área de la aurícula izquierda (AAI), la masa ventricular izquierda (MVI), el índice de masa ventricular izquierda (IMVI), el grosor relativo (GR), la fracción de eyección (FE), la fracción de acortamiento (FA) y la presencia de derrame pericárdico (DP). La evaluación funcional se hizo durante las dos a tres semanas siguientes del posoperatorio y previo al alta hospitalaria. En la mayoría de los pacientes se realizó una prueba de ejercicio convencional utilizando los protocolos de Bruce y Bruce modificado aplicados de acuerdo con el estado del paciente. Esta evaluación fue realizada en el Departamento de Medicina Física y Rehabilitación del HUSVP por dos médicos rehabilitadores con experiencia reconocida en el campo de la rehabilitación cardiaca. Los resultados de la prueba fueron medidos en METS y se registraron en el formulario como estado funcional (17). Uno de los investigadores se encargó de evaluar personalmente o por vía telefónica periódicamente a los pacientes para no perder el contacto y conocer su evolución. Cada uno de los pacientes que se vinculó en el estudio fue evaluado a los seis meses, con un examen clínico, un ecocardiograma transtorácico y una evaluación funcional, los cuales fueron realizados por el mismo grupo de médicos y bajo las mismas especificaciones y parámetros que en el momento de la hospitalización.

Para el manejo y organización de datos se construyó una base de datos en el programa Excel 2000 y en SPSS Versión 9 , en la cual se tuvieron en cuenta controles para el chequeo de la entrada de datos y su verificación.

Se realizó un análisis univariado para describir en ambos grupos las características epidemiológicas de la lesión, el tipo de cirugía, las complicaciones, los hallazgos EKG, ecocardiográficos y de la prueba de ejercicio en la primera evaluación.

Se realizó un análisis bivariado para establecer las diferencias en las variables entre los grupos así: diferencias entre la cohorte expuesta y la no expuesta en la evaluación inicial y a los seis meses; diferencias en el primer grupo entre la evaluación inicial y a los seis meses; diferencias en el segundo grupo entre la evaluación inicial y a los seis meses;diferencias en cada una de las mediciones en cada individuo al principio y a los seis meses (diferencia intrapaciente); promedio de las diferencias en la cohorte expuesta y la no expuesta; comparación de los promedios de las diferencias.

Para determinar si las variables cuantitativas tienen una distribución normal se realizó una prueba de Shapiro Wilks, si las variables eran normales se hacía una diferencia de promedios. Para evaluar su significancia estadística se utilizó en el caso de comparar la primera con la segunda evaluación una $t$ de student $t$ pareada o cuando se comparó entre grupos una $t$ student para muestras independientes. Si las variables tenían un comportamiento no normal se tomaron las medianas con sus rangos intercuartiles y se aplicó la prueba $\mathrm{U}$ de Mann Whitney para probar estadísticamente las diferencias. Para las variables nominales se determinaron las proporciones y se analizaron mediante una prueba Chi cuadrado. 
Para el análisis de la asociación se tomó como exposición tener el infarto de miocardio y como desenlace la remodelación cardiaca medida por el índice de masa ventricular izquierdo y por el grosor relativo de pared (IMVI mayor a $125 \mathrm{~g} / \mathrm{m}^{2}$ en hombres y $110 \mathrm{~g} / \mathrm{m}^{2}$ en mujeres y GR igual o menor de 0,45$)$. Se calculó la incidencia en los expuestos y en los no expuestos, con lo cual se estableció el riesgo relativo con su respectivo intervalos de confianza.

Para el análisis se utilizó el paquete estadístico SPSS versión 9.

\section{Resultados \\ Características epidemiológicas}

Entre enero de 2002 y abril de 2004 se evaluaron 103 pacientes con herida penetrante de corazón. En este informe se presentan los resultados de 33 pacientes que cumplieron con los criterios de inclusión. En 11 de estos pacientes se documentó infarto de miocardio y conforman el grupo expuesto; los restantes 22 integran el grupo no expuesto.

Las características epidemiológicas de los pacientes se presentan en la Tabla 1. La mayoría de los pacientes eran hombres jóvenes procedentes del área urbana de los municipios del Valle de Aburrá. Los antecedentes más comunes fueron el consumo de licor y de cigarrillo, que fueron mayores en el grupo expuesto. El consumo de drogas ilícitas fue muy similar en ambos grupos 63.6\% y 54.5\%; las principales drogas referidas por los pacientes fueron la marihuana, el bazuco y la cocaína. La mayoría de los pacientes evaluados eran sedentarios, sólo $27.3 \%$ de los pacientes del grupo con infarto de miocardio y $18.2 \%$ en el grupo sin infarto realizaban actividad física regular. Seis pacientes en el grupo de infarto de miocardio recibieron medicamentos que potencialmente modulan el proceso de

Tabla 1. Características clínicas y epidemiológicas de los pacientes que sufrieron heridas de corazón con y sin infarto traumático.

\begin{tabular}{|l|c|c|}
\hline & $\begin{array}{c}\text { Infarto } \\
\text { Traumático=11 } \\
\mathbf{\%}\end{array}$ & $\begin{array}{c}\text { Sin infarto } \\
\mathbf{n = 2 2} \\
\mathbf{\%}\end{array}$ \\
\hline Edad & $31.1(14)^{*}$ & $31.7(12.8)^{*}$ \\
Sexo masculino & 90.9 & 95.5 \\
Población urbana & 100 & 95.5 \\
Desempleados & 45.5 & 36.4 \\
Tipo de arma & & \\
$\quad$ Arma de fuego & 9.1 & 4.5 \\
Arma cortopunzante & 90.9 & 95.5 \\
Localización de la herida & & 45.5 \\
Ventrículo derecho & 36.4 & 22.7 \\
Ventrículo izquierdo & 63.6 & 31.8 \\
Aurícula & & 31.8 \\
Otras heridas & 27.3 & - \\
Complicaciones & & 45.5 \\
Falla cardiaca & & 9.1 \\
Infección & 27,3 & \\
Otras & 54.5 & \\
\hline * Media y desviación estándar & 27.3 & \\
\hline
\end{tabular}

remodelación por indicación de su médico tratante, aunque sólo uno de ellos lo hizo en forma regular durante los seis meses de seguimiento. El 27.2\% de los pacientes con infarto de miocardio traumático desarrollaron síndrome de falla cardiaca, requiriendo manejo especifico intrahospitalario en dos de ellos.

\section{Características clínicas}

Las características clínicas se presentan en la Tabla 1. De acuerdo con la descripción operatoria, el ventrículo derecho y el ventrículo izquierdo fueron las estructuras cardiacas más comprometidas, todos los pacientes del grupo expuesto y $68.2 \%$ del grupo no expuesto presentaron compromiso de estas cavidades. En uno de los pacientes con infarto de miocardio se encontró lesión directa de la circulación coronaria. En los pacientes del grupo expuesto la extensión del infarto principalmente fue de la pared apical (27.2\%), seguido por la pared anterior y la anterolateral. En dos de los pacientes con infarto traumático se realizó cateterismo.

Ningún paciente del grupo no expuesto presentó isquemia miocárdica en el posoperatorio. Tres de los pacientes del grupo expuesto presentaron signos de falla cardiaca durante el seguimiento, dos con un IM anterolateral extenso con falla cardiaca en forma temprana y en quien los médicos tratantes iniciaron medicamentos con efecto en la remodelación; sin embargo, sólo uno de ellos tomó la medicación durante los seis meses de seguimiento. La otra paciente fue una mujer farmacodependiente quien al asistir al control de los seis meses se encontraba en embarazo. Esta paciente presentó signos de falla cardiaca, por lo cual requirió hospitalización durante el tercer trimestre del embarazo.

En seis de los pacientes con infarto (54.5\%), el grupo tratante prescribió medicamentos con potencial de remodelación cardiaca al momento del alta hospitalaria; sin embargo, solamente un paciente continuó tomando la medicación durante los seis meses y asistió a controles mensuales con su médico tratante para realizar los ajustes necesarios. En el momento de la evaluación a los seis meses este paciente recibía captopril $75 \mathrm{mg} /$ día, metoprolol $100 \mathrm{mg} /$ día, betametildigoxina $0.1 \mathrm{mg} /$ día y espirinolactona $40 \mathrm{mg} /$ día. Los otros cinco pacientes comentaron en la evaluación de los seis meses que habían tomado los medicamentos en forma irregular y los habían suspendido.

En dos $(9.1 \%)$ de los pacientes sin infarto, se prescribieron medicamentos con potencial de remodelación cardiaca, al primero de ellos se encontró hipertenso y se prescribió propanolol $80 \mathrm{mg} /$ día. El segundo recibió captopril $75 \mathrm{mg} /$ día durante un mes, tras el cual el paciente decidió suspenderlo.

\section{Características ecocardiográficas y evaluación funcional}

La Tabla 2 presenta las características ecocardiográficas y la prueba funcional al inicio y a los seis meses, en los grupos con y sin infarto. La fracción de eyección y la fracción de acortamiento fueron significativamente menores en los 
Tabla 2. Características ecocardiográficas y capacidad funcional iniciales y luego de seis meses de seguimiento.

\begin{tabular}{|c|c|c|c|c|c|c|}
\hline \multirow[b]{2}{*}{ Parámetro } & \multicolumn{3}{|c|}{ Sin infarto traumático } & \multicolumn{3}{|c|}{ Con infarto traumático } \\
\hline & $\begin{array}{c}\text { Inicial } \\
\mathbf{n}=11\end{array}$ & $\begin{array}{l}6 \text { Meses } \\
n=11\end{array}$ & Valor de $p$ & $\begin{array}{c}\text { Inicial } \\
\mathrm{n}=11\end{array}$ & $\begin{array}{l}6 \text { Meses } \\
n=11\end{array}$ & Valor de $p$ \\
\hline METS & $4(2-8)^{\mathrm{a}}$ & $10(3-18)^{\mathrm{a}}$ & 0.004 & $5(3-10)^{a}$ & $13(7-17)^{\mathrm{a}}$ & 0.000 \\
\hline $\operatorname{LVDD}(\mathrm{cm})$ & $4.78(0.34)$ & $5.12(0.67)$ & 0.130 & $4.69(0.47)$ & $4.91(0.35)$ & 0.020 \\
\hline $\operatorname{LVSD}(\mathrm{cm})$ & $3.19(0.48)$ & $3.46(0.74)$ & 0.242 & $2.97(0.54)$ & $3.15(0.39)$ & 0.165 \\
\hline $\operatorname{RAA}\left(\mathrm{cm}^{2}\right)$ & $14.27(1.79)$ & $14.46(6.44)$ & 0.932 & $13.81(2.61)$ & $14.19(2.84)$ & 0.709 \\
\hline $\operatorname{LAA}\left(\mathrm{cm}^{2}\right)$ & $15.64(2.91)$ & $14.09(5.19)$ & 0.395 & $13.74(2.49)$ & $12.76(2.68)$ & 0.160 \\
\hline LVM (g) & $179.83(33.4)$ & $173.09(40.2)$ & 0.652 & $176.42(39.7)$ & $164.1(34.6)$ & 0.234 \\
\hline $\operatorname{LVMI}\left(\mathrm{g} / \mathrm{m}^{2}\right)$ & $99.42(20.3)$ & $107.5(28.3)$ & 0.283 & $103.62(20.5)$ & $99.16(20.4)$ & 0.374 \\
\hline RT & $0.45(0.08)$ & $0.38(0.08)$ & 0.010 & $0.46(0.08)$ & $0.39(0.05)$ & 0.002 \\
\hline $\mathrm{EF} \%$ & $52(40-65)^{\mathrm{a}}$ & $60(30-75)$ & 0.339 & $70(55-75)^{\mathrm{a}}$ & $65(50-75)$ & 0.264 \\
\hline $\mathrm{SF} \%$ & $27.63(5.78)$ & $30.18(8.38)$ & 0.343 & $35.1(4.54)$ & $34.57(4.3)$ & 0.736 \\
\hline \multicolumn{7}{|c|}{$\begin{array}{l}\text { METS: equivalente metabólico } 1 \mathrm{MET} \text { equivale a } 3.5 \mathrm{ml} \mathrm{O} / \mathrm{kg} / \mathrm{min} \text {; LVDD: L diámetro en fin de diástole; LVSD: diámetro en fin de sístole; RAA: área aurícula derecha; } \\
\text { LAA: área auricular izquierdo; LVM: masa ventricular izquierda; LVMI: índice de masa ventricular izquierda; RT: grosor relativo; EF: fracción de expulsión; ST: fracción de acortamien }\end{array}$} \\
\hline
\end{tabular}

Tabla 3. Características ecocardiográficas y funcionales luego de seis meses de seguimiento.

\begin{tabular}{|c|c|c|c|}
\hline Características & $\begin{array}{c}\text { Sin infarto } \\
\text { traumático } \\
(\mathbf{n}=11)\end{array}$ & $\begin{array}{c}\text { Con infarto } \\
\text { traumático } \\
(\mathrm{n}=22)\end{array}$ & Valor de $p$ \\
\hline METS & $10(3-18)^{\mathrm{a}}$ & $13(7-17)^{\mathrm{a}}$ & 0.191 \\
\hline $\operatorname{LVDD}(\mathrm{cm})$ & $5.12(0.67)$ & $4.91(0.35)$ & 0.251 \\
\hline $\operatorname{LVSD}(\mathrm{cm})$ & $3.46(0.74)$ & $3.13(0.39)$ & 0.100 \\
\hline $\operatorname{RAA}\left(\mathrm{cm}^{2}\right)$ & $14.46(6.44)$ & $13.5(2.84)$ & 0.556 \\
\hline $\operatorname{LAA}\left(\mathrm{cm}^{2}\right)$ & $14.09(5.19)$ & $12.5(2.44)$ & 0.236 \\
\hline MVI. (g) & $173.09(40.16)$ & $164.45(33.78)$ & 0.520 \\
\hline LVMI (g/m²) & $107.50(28.33)$ & 99.41 (19.99) & 0.349 \\
\hline RT & $0.38(0.084)$ & $0.39(0.05)$ & 0.445 \\
\hline $\mathrm{EF}(\%)$ & $60(30 \text { a } 75)^{\mathrm{a}}$ & $65(50 \text { a } 75)^{\mathrm{a}}$ & 0.191 \\
\hline $\mathrm{SF}(\%)$ & $30.18(8.38)$ & 34.73 (4.26) & 0.046 \\
\hline \multicolumn{4}{|c|}{$\begin{array}{l}\text { METS: equivalente metabólico, } 1 \text { MET equivale a } 3.5 \mathrm{ml} \mathrm{O} / \mathrm{kg} / \mathrm{min} \text {; LVDD: diámetro } \\
\text { ventricular en fin de diástole; LVSD: diámetro ventricular en fin de sístole; RAA: área } \\
\text { auricular derecha; LAA: área auricular izquierda; LVM: masa ventricular izquierda; } \\
\text { LVMI: índice de masa ventricular izquierda; RT: grosor relativo; EF: Fracción de } \\
\text { expulsión; ST: fracción de acortamiento. }\end{array}$} \\
\hline \multicolumn{4}{|c|}{ a Media, la significacia estadística se calculó utilizando el test de Student } \\
\hline
\end{tabular}

pacientes infartados. La mediana de la fracción de eyección en éstos fue de 52, mientras que en los no infartados fue de 70. Por tanto, en los pacientes con infarto se encontró compromiso de la función sistólica en las etapas iniciales. La capacidad funcional fue menor en los infartados, pero esto no fue estadísticamente significativo. Para el resto de variables no hubo diferencias significativas.

La Tabla 3 presenta las características ecocardiográficas y la prueba funcional a los seis meses de seguimiento en ambos grupos. En ese momento, los valores del DDVI, el
Tabla 4. Diferencias intrapaciente en las variables ecocardiográficas.

\begin{tabular}{|l|c|c|c|}
\hline Características & $\begin{array}{c}\text { Sin infarto } \\
(\mathbf{n = 1 1})\end{array}$ & $\begin{array}{c}\text { Con infarto } \\
(\mathbf{n = 2 2})\end{array}$ & Valor de p \\
\hline LVDD $(\mathrm{cm})$ & 0.34 & 0.23 & 0.560 \\
LVSD $(\mathrm{cm})$ & 0.27 & 0.18 & 0.712 \\
RAA $\left(\mathrm{cm}^{2}\right)$ & 0.18 & 0.38 & 0.927 \\
LAA $\left(\mathrm{cm}^{2}\right)$ & -1.55 & -0.97 & 0.724 \\
LVM $(\mathrm{g})$ & -6.74 & -12.33 & 0.754 \\
LVMI $\left(\mathrm{g} / \mathrm{m}^{2}\right)$ & 8.07 & -4.46 & 0.151 \\
RT & -0.075 & -0.068 & 0.840 \\
SF \% & 2.55 & -0.524 & 0.283 \\
\hline $\begin{array}{l}\text { LVDD: diámetro ventricular en fin de diástole; LVSD: diámetro ventricular en fin } \\
\text { de sístole; RAA: área auricular derecha; LAA: área auricular izquierda; LVM: masa }\end{array}$ \\
ventricular izquierda; LVMI: índice de masa ventricular izquierda; RT: grosor relativo; \\
$\begin{array}{l}\text { EF: fracción de expulsión; ST: fracción de acortamiento. Se utilizó la prueba T para } \\
\text { muestras independientes. }\end{array}$
\end{tabular}

Tabla 5. Incidencia de la remodelación cardiaca en pacientes con herida penetrante de corazón con y sin infarto traumático.

\begin{tabular}{|l|c|c|c|c|}
\hline \multirow{2}{*}{ Grupo } & \multicolumn{2}{|c|}{$\begin{array}{c}\text { Número de pacientes } \\
\text { con remodelamiento* }\end{array}$} & \multirow{2}{*}{ Total } & \multirow{2}{*}{ Incidencia } \\
\cline { 2 - 3 } & Hombres & Mujeres & & \\
\hline Con infarto & 3 & 1 & 11 & 0.36 \\
Sin infarto & 3 & 22 & 0.14 & \\
Total & & & 23 & \\
Riesgo relativo & Intervalo de confianza & & $(0.72-9.89)$ & \\
\hline $\begin{array}{l}\text { *Pacientes con índice de masa ventricular mayor a } 125 \mathrm{~g} / \mathrm{m}^{2} \text { en hombres y } 110 \mathrm{~g} / \mathrm{m}^{2} \\
\text { en mujeres. }\end{array}$ \\
\hline
\end{tabular}


DSVI y el IMVI fueron más altos en el grupo con infarto pero sin significancia estadística. El GR y la FE fueron menores en el grupo con infarto, pero sin significancia estadística. La única variable con diferencia significativa entre los dos grupos fue la fracción de acortamiento (30.18 Vs 34.72, p < 0.05). La capacidad funcional alcanzada por los pacientes sin infarto fue superior a la lograda por los con infarto.

Se observa un incremento en el DDVI, en el DSVI y el IMVI, pero sin que sea estadísticamente significativo. El GR disminuyó en forma estadísticamente significativa ( $\mathrm{p}<0.05)$. La FE y la FA mejoraron sin significancia estadística. El estado funcional incrementó en forma significativa.

En los pacientes del grupo sin infarto se observa un incremento significativo en el DDVI y un incremento no significativo del DSVI. El IMVI y la FE disminuyeron en forma no significativa. El GR disminuyó significativamente. $(\mathrm{p}<0.05)$. La capacidad funcional mejoró en forma importante $(\mathrm{p}<0.05)$.

La Tabla 4 presenta la media de las diferencias para cada una de las variables que siguen una distribución normal en los pacientes con y sin infarto. La media de diferencias para el DDVI y el DSVI fue mayor en el grupo expuesto con tendencia al incremento en los diámetros. El IMVI en los expuestos se incrementó y la media de las diferencias fue muy importante en comparación con la del grupo no expuesto (+8.07 Vs -4.46). El GR disminuyó en ambos grupos, pero la media de las diferencias fue mayor en el grupo expuesto. Sin embargo, para ninguna de las variables evaluadas se encontraron diferencias estadísticamente significativas entre los dos grupos.

Finalmente en la Tabla 5 se observa que los pacientes con herida penetrante de corazón en quienes se documenta infarto de miocardio secundario tienen un RR de 2.67 (0.729.89) de desarrollar hipertrofia excéntrica a los seis meses que aquellos que no presentan infarto. Como se observa el intervalo de confianza es muy amplio e incluso atraviesa el valor de 1 por lo cual no es concluyente. Esto es debido al tamaño de muestra.

\section{Discusión}

El infarto agudo de miocardio traumático y en general las lesiones traumáticas del corazón se presentan en poblaciones con condiciones socioeconómicas y culturales pobres. La mayoría de ellos son hombres jóvenes, sedentarios, con edades entre los 17 a los 45 años, desempleados o con pobre estabilidad laboral y con historia de farmacodependencia en su mayoría, por lo que el seguimiento a largo plazo se hace particularmente difícil y los datos al respecto son escasos (1-7).

Más de $90 \%$ de las heridas fueron ocasionadas por arma cortopunzante, muy similar a lo encontrado por Duque y colaboradores; sin embargo, cuando se revisa la literatura sobre herida penetrante de corazón se encuentra un predominio mayor del proyectil de arma de fuego como causante de las lesiones. Esto puede explicarse porque esta cohorte son los que sobrevivieron a la herida inicial, la mortalidad en pacientes heridos con proyectil de arma de fuego es alta y la mayoría de ellos fallecen antes de recibir atención hospitalaria $(3,6,7)$. Otro mecanismo importante, reportado en la literatura como causa de infarto, es el trauma cerrado de tórax y la contusión miocárdica (31-34).

El ventrículo izquierdo es la cámara más frecuentemente afectada, seguido por el ventrículo derecho, lo cual está de acuerdo con lo informado en la literatura y con lo reportado por Demetriades y Mattox (1-8). Todos los pacientes con infarto presentaron compromiso del ventrículo izquierdo y/o el derecho. La extensión del infarto fue principalmente apical, seguido por la pared anterior y anterolateral, este hecho también puede generar un proceso de remodelación adverso de acuerdo con lo informado en la literatura. La arteria descendente anterior fue la responsable del infarto en dos pacientes a quienes se realizó cateterismo; sin embargo, no fue posible determinar el mecanismo exacto de la lesión.

Durante la hospitalización, las variables ecocardiográficas evaluadas fueron similares para ambos grupos, con excepción de la fracción de eyección y de acortamiento, que fueron significativamente menores en el grupo expuesto, lo que indica que estos pacientes tuvieron un compromiso miocárdico importante que deterioró la función sistólica. Durante el seguimiento los pacientes con infarto traumático exhiben una tendencia al desarrollo de un patrón de hipertrofia excéntrica como mecanismo compensatorio, con aumento del índice de masa ventricular izquierda, de los diámetros ventriculares y disminución del grosor relativo, lo que permite preservar la función ventricular sistólica evaluada como fracción de eyección a corto plazo. En forma interesante, los pacientes con herida penetrante sin infarto de miocardio, presentan un patrón similar con incremento de los diámetros ventriculares y disminución del grosor relativo; sin embargo, no existe tendencia a aumentar el IMVI; ésto sugiere la inducción de remodelación ventricular, que por no estar acompañada de hipertrofia y aumento de tensión miocárdica, podría generar en menor grado síndrome de falla cardiaca (35). Hasta el momento se desconoce si este patrón ha sido reportado en otros estudios, en pacientes con herida penetrante de corazón sin infarto. Este hallazgo ha sido mencionado por otros investigdores, quienes encontraron que el incremento en la dimensión interna del ventrículo izquierdo, en población sin historia de infarto o trauma, es un factor de riesgo para falla cardiaca congestiva en hombres y mujeres quienes no han tenido un infarto de miocardio (8). A pesar de la demostración de un patrón claro de remodelación ventricular, las diferencias son pequeñas debido al número de pacientes y al corto tiempo de seguimiento.

Al comparar los dos grupos a los seis meses de seguimiento, se encontró diferencia significativa en la fracción de acortamiento (FA), de $30.18 \mathrm{Vs} 34.73$, grupo expuesto Vs no expuesto, respectivamente y $\mathrm{p}<0.05$. La FA es un índice ampliamente utilizado de la función sistólica, medido 
en los trazos del modo $\mathrm{M}$ y particularmente útil, por cuanto el acortamiento de las fibras miocárdicas es responsable de la mayoría del volumen sistólico (31). Desde este punto de vista la FA es similar a la FE y en ausencia de alteraciones segmentarias en el movimiento de la pared, los dos índices se correlacionan estrechamente. Tanto la FE como la FA son determinadas por otros factores, además de la función contráctil y estos índices deben interpretarse teniendo en cuenta las condiciones de carga, pues el incremento en la precarga y la disminución en la poscarga pueden incrementar la FA y pueden ser operador dependiente $(31,32)$.

El DDVI, el DSVI y el IMVI fueron mayores en los pacientes con infarto. El GR, la FE y la capacidad funcional fueron menores en los expuestos. Sin embargo, para ninguna de estas variables se alcanzó diferencia estadística significativa. Además, también se calculó la media de las diferencias para cada variable en cada grupo y se demostró que para los diámetros, el IMVI y el GR la media de las diferencias siempre fue mayor en el grupo expuesto en favor de la dilatación y la hipertrofia.

Por último, se evaluó el peor escenario de la remodelación adversa del ventrículo izquierdo en ambos grupos y calculamos la incidencia de hipertrofia excéntrica en cada grupo. El RR fue 2.67 (0.72-9.89), lo que indica que los pacientes de este estudio con herida penetrante de corazón e infarto de miocardio secundario desarrollaron hipertrofia excéntrica del ventrículo izquierdo. El intervalo de confianza (IC 95\% 0.72-9.89) es poco preciso e incluye el 1; lo anterior fue debido al poder de $80 \%$ y se hubieran necesitado 135 pacientes.

La fracción de eyección en el posinfarto inmediato es un factor predictor de desarrollo de síndrome de falla cardiaca a corto plazo (36). En el grupo de pacientes con infarto traumático $27.2 \%$ de los pacientes desarrollaron el síndrome en contraposición al grupo no expuesto, en el que no se demostraron manifestaciones clínicas sugestivas de disfunción ventricular sistólica.

\section{Limitaciones del estudio}

Son bien conocidas las dificultades socioeconómicas y culturales que presentan los pacientes con heridas penetrantes de corazón, por lo cual su seguimiento a largo plazo es imposible. Es probable que en un tiempo tan corto de seguimiento, seis meses, no se observen alteraciones significativas desde el punto de vista estadístico en los parámetros evaluados, sin embargo esto no impide observar la tendencia demostrada en el estudio.

El mecanismo exacto del infarto de miocardio no se determinó, debido a que las condiciones clínicas iniciales de la mayoría de los pacientes no permiten la realización de estudios específicos, como angiografía coronaria. Es posible que la presencia de enfermedad coronaria concomitante y/o circulación colateral altere los resultados, aunque por la edad de la población estudiada su probabilidad es baja. Existen métodos diagnósticos, como la resonancia magnética nuclear, que ofrece ventajas sobre la evaluación ecocardiográfica; sin embargo, las condiciones iniciales mencionadas de este grupo de pacientes impiden su utilización rutinaria.

Dado que no es un estudio de intervención, no se controló la administración de medicamentos que afectan en forma positiva la remodelación ventricular y la decisión estuvo a consideración del grupo médico tratante y es probable que se extrapole el beneficio de los IECA, betabloqueadores y otros medicamentos a los pacientes con infarto traumático (36). Si bien un porcentaje importante de pacientes expuestos recibió algunos de estos medicamentos, sólo uno lo hizo en forma continua durante los seis meses y el resto por menos de dos meses.

En último lugar, los pacientes no expuestos desarrollan también remodelación ventricular, lo que implicaría un tamaño de muestra mayor que el calculado inicialmente para lograr establecer diferencias significativas entre los dos grupos.

\section{Conclusiones}

Los pacientes con herida penetrante de corazón con y sin infarto presentaron tendencia a realizar dilatación del ventrículo izquierdo, la cual se manifestó por un incremento en los diámetros ventriculares y una reducción en el grosor relativo; los pacientes con infarto tienen tendencia a realizar una mayor dilatación ventricular comparado con los no infartados. Sin embargo, las diferencias encontradas no son significativas y se requiere de un seguimiento más largo e incrementar el número de pacientes.

\section{Referencias}

1. Cawford FA. Heridas penetrantes del corazón. Sabiston DC. Tratado de Patología Quirúrgica. México. 14a . edición. Interamericana McGraw-Hill; 1991.p. 2060-5.

2. Cohn, PE, Braunwald E. Traumatic Heart disease. Braunwald E. Heart Disease 4a. edición. WB Saunders; 1992. p. 1517-27.

3. Duque HA, Flórez LE, Moreno A, Jurado H, Jaramillo CJ, Restrepo MC. Penetrating cardiac trauma follow-up Study including Electrocardiography, Ecocardiography and Functional test. World J Of Surg 1999; 23: 1254-7.

4. Asensio J, Berne JD, Demetriades D, Chan L, Murria J, Falabella A. et al. One hundred five Penetrating Cardiac Injuries: A 2-year prospective evaluation. The J of Trauma 1998; 44: 1073-82.

5. Demetriades D, Veen BW. Penetrating injuries of the Heart: Experience over two years in South Africa. J of Trauma 1983; 23: 1034-41.

6. Tibursky JG, Astra L, Wilson RF, Dente C, Steffes C. Factors Affecting prognosis with penetrating Wounds of the heart. J of Trauma. 2000; 48: 587-91.

7. Camphell NC. Review of 1198 cases of penetrating cardiac trauma. Br J Surg 1997; 84: 1737.

8. Demetriades D, Charalambides C, Sareli P, Pantanowitz D. Late sequelae of penetrating cardiac injuries. Br J Surg 1990; 77: 813-4.

9. Weber KT, Janicki JS. Pathogenesis of Heart Failure. Card Clin 1989; 7: 11-23.

10. Bologuese L, Cerisano G. Early predictors of left ventricular remodeling after acute myocardial infarction. Am Heart J 1999; 138: 579-83.

11. Symbas PN, Arensberg D. Traumatic heart desease. Hurst JW. Schlant RC. The Heart. McGraw-Hill. 1990. Seventh edition. 1375-81.

12. Mattox KL, Limacher MC, Feliciano DV, Colsimo L, O'meara ME, Beall AC. et al. Cardiac Evaluation Following Heart Injury. J of Trauma Aug 1985; 25: 758-65.

13. Pinilla AA, Mora M, Ramírez J, Patiño JF, Garcia E, Cruz AV. Heridas de corazón (informe preliminar). Rev Col Anest 1979; 7: 78.

14. Cha EK, Mittal V, Allaben RD. Delayed sequelae of penetrating cardiac injury. Arch Surg 1993; 128: 636-9. 
15. Fallah-Nejad M, Wallace HW, Su CC, Kutty AC, Blakemore WS. Unusual manifestations of penetrating cardiac injuries. Arch Surg 1975; 110: 1357-62.

16. Heller RF, Rahimttla SH, Ehsani A, Johnson S, Boyd DR, Tatooles CJ. Cardiac complications: Results of penetrating chest injuries involving the heart. Arch Intern Med 1974; 134: 491.

17. Moldover JR, Bartels MN. Cardiac Rehabilitation. Braddom RL. Physical Medicine and Rehabilitation. WB Saunders. 1996. P. 649-69.

18. Reissman P, Rivkind A, Jurim O, Simon D. Case report: The management of penetrating cardiac trauma with major coronary injury - is cardiopulmonary bypass essential? J Trauma 1992; 33: 773-5.

19. Willenheimer R. Left ventricular remodeling and dysfunction. Can the process be prevented?. Int J Cardiol 2000; 72: 143-50.

20. Sonnenblick EH, Anversa P. Models and remodeling: mechanism and clinical implications. Cardiologia 1999; 44: 609-19.

21. Pfeffer MA. Ventricular remodeling and expansion after Myocardial infarction. Acute Myocardial Infarction. Elsevier Science Publishing. New York. 1991. P. 438-48.

22. Sanchis J, Bodi V, Insa LD, Berenguer A. Chorro FJ, Llacer A. et al. Predictors of early and late ventricular remodeling after acute myocardial infarction. Clinic Cardiol Sept 1999; 22: 581-6.

23. Dubach P., Myers J, Dzickan G, Goebbels U, Reinhart W, Vugt P. Effect of Exercise Training on Myocardial Remodeling in patients with reduced left ventricular function after myocardial infarction. Circulation 1997; 95: 2060-6.

24. Devereux RB, Reichek N. Echocardiographic determination of left ventricular mass in men. Anatomic validation of the method. Circulation 1977; 55: 613-8.

25. Devereux RB, Alonso DR, Lutas EM. Echocardiographic assesement of left ventricular hipertrophy: comparison to nrcropsy findings. Am J Cardiol 1986; 57: 450-8.
26. Koren MJ, Devereux RB, Casale PN, Savage DD, Laragh, JH. Relation of Left Ventricular Mass and Geometry to Morbidity and Mortality in Uncomplicated Essential Hypertension. Annals of Internal Medicine 1991; 114: 345-51.

27. Antman EM, Braunwald E. Acute myocardial infarction. Harrison's. Principes of Internal Medicine. Editorial McGraw-Hill.2001. Edición 15. Cap 243.

28. Jugdutt BI. Ventricular Remodeling after infarction and the Extracellular Collagen Matriz. When is enough enough?. Circulation 2003, 108: 1395-403.

29. Garg S, Narula J. Pathogenetic basis of myocardial dysfunction and amenability to reversal. Cardiol clinic 2003; 21: 83-91.

30. Alpert JS, Thygesen K, Jaffe A, White HD. The universal definition of myocardial infarction: a consensus document: ischaemic heart disease. Heart. 2008; 94: $1335-41$.

31. Aurigemma GP, Douglas PS, Gaasch WH. Quantitative Evaluation of Lef Ventricular structure, wall stress, and systolic function. Otto $\mathrm{CH}$. The practice of clinical echocardiography 2a. Edición. WB Saunders company; 2002. p 65-87.

32. Sheehan FH. Quantitative Evaluation of regional left ventricular Systolic function Otto $\mathrm{CH}$. The practice of clinical echocardiography 2a. Edición. WB Saunders company; 2002.p 88-112

33. Ismailov R, Ness $\mathbf{R}$, Weiss $\mathbf{H}$ et al. Trauma associated with acute myocardial infarction in a multistated hospitalized population. Int J Cardiol 2005; 105: 141-6.

34. Rodrigues A, Furlanetti L, Faidiga G, et al. Penetrating cardiac injuries: a 13 years retrospective evaluation from a Brazilian trauma center. Int Cardiovasc Thorac Surg 2005; 4: 212-5.

35. Bohm M, Kilter H, Kindermann M. Mechanisms contributing to the progression of left ventricular dysfunction to end-stage heart failure. Eur Heart J 2003; 5 (suppl I):114-21.

36. Cohn J, Ferrari R, Sharpe N. From behalf on International Forum on Cardiac Remodelling. J Am Coll Cardiol 2000; 35: 569-82. 